\title{
Stable rationality and conic bundles
}

Hassett, Brendan ; Kresch, Andrew ; Tschinkel, Yuri

Abstract: We study stable rationality properties of conic bundles over rational surfaces.

DOI: https://doi.org/10.1007/s00208-015-1292-y

Posted at the Zurich Open Repository and Archive, University of Zurich ZORA URL: https://doi.org/10.5167/uzh-128400

Journal Article

Accepted Version

Originally published at:

Hassett, Brendan; Kresch, Andrew; Tschinkel, Yuri (2016). Stable rationality and conic bundles. Mathematische Annalen, 365(3-4):1201-1217.

DOI: https://doi.org/10.1007/s00208-015-1292-y 


\title{
STABLE RATIONALITY AND CONIC BUNDLES
}

\author{
BRENDAN HASSETT, ANDREW KRESCH, AND YURI TSCHINKEL
}

ABSTRACT. We study stable rationality properties of conic bundles over rational surfaces.

\section{INTRODUCTION}

Let $k$ be an algebraically closed field, $X$ a smooth projective variety over $k$. We say $X$ is stably rational if $X \times \mathbb{P}^{n}$ is rational for some $n \in \mathbb{N}$. A breakthrough in the problem of addressing the stable rationality of varieties was achieved by Voisin [45], who gave a remarkably powerful sufficient criterion for the very general member of a family of varieties to fail to be stably rational. Working over the complex numbers, she shows that the double cover of $\mathbb{P}^{3}$ branched along a very general quartic surface is not stably rational. The degeneration aspects of Voisin's method were generalized and simplified by Colliot-Thélène and Pirutka and applied to quartic threefolds and to cyclic covers of projective space of prime degree $[18,19]$. These techniques have also been applied to families of double covers by Beauville [11, 12]; using a mixed characteristic version, introduced in [18], and an idea of Kollár [26], Totaro proved that many hypersurfaces in $\mathbb{P}^{n}$ are not stably rational [43].

The problem of rationality of conic bundles has a long history; see, e.g., [9] [25, Conjecture I] [40]. See also [42, Main Thm.] for rationality criteria.

An obvious necessary condition for (stable) rationality is unirationality. We recall, $X$ is unirational if there exists a dominant rational map from a projective space to $X$. In characteristic zero, some cases of unirational conic bundles are identified in [31], e.g., those with smooth branch locus in $\mathbb{P}^{2}$ of degree $\leq 8$.

In this paper we address the question of stable rationality of conic bundles over rational surfaces. Our main result is:

Theorem 1. Let $S$ be a rational smooth projective surface over an uncountable algebraically closed field $k$ of characteristic different from 2 . Let $\mathcal{L}$ be a linear system of effective divisors on $S$ whose general member is smooth and irreducible. Let $\mathcal{M}$ be an irreducible component of the space of reduced nodal curves in $\mathcal{L}$ together with degree 2 étale covering. Assume that $\mathcal{M}$ contains a cover, nontrivial over every irreducible component of a reducible curve with smooth irreducible components and set of nodes disjoint from the base locus of $\mathcal{L}$. Then the conic bundle over $S$ corresponding to a very general point of $\mathcal{M}$ is not stably rational.

By [7, Thm. 1] and [41, Thm. 5.7], for each point $\left\{D^{\prime} \rightarrow D\right\} \in \mathcal{M}$ there is a conic bundle over $S$ with discriminant curve $D$; this is defined up to birational isomorphism. We remark that covers acquiring ramification above the nodes are excluded from $\mathcal{M}$.

For example, Theorem 1 is applicable to the complete linear system of degree $d$ curves in $\mathbb{P}^{2}$ for $d \geq 6$. A similar analysis is applicable, e.g., to Hirzebruch surfaces.

Date: August 4, 2015. 
(See $[10,24]$ for analysis of the monodromy of two-torsion points of Jacobians of curves in rational ruled surfaces.) Our method is not applicable when the discriminant is a plane quintic or a tri-section of a rational ruled surface; reducible curves of these types necessarily have rational components.

Acknowlegdments: The first author was supported by NSF grants 1148609 and 1401764. The second author was supported by the Swiss National Science Foundation. The third author was supported by NSF grant 1160859. We are grateful to I. Cheltsov, J.-L. Colliot-Thélène, L. Katzarkov, A. Pirutka, and B. Totaro for comments and suggestions.

\section{BACKGROUND}

2.1. Brauer group. The Brauer group, for us, is the cohomological Brauer group

$$
\operatorname{Br}(S):=H^{2}\left(S, \mathbb{G}_{m}\right)_{\text {tors }}
$$

of a Noetherian scheme or Deligne-Mumford stack $S$. (Here and elsewhere we work with étale cohomology groups.) The role of points and residue fields of a scheme is played by residual gerbes

$$
f_{\xi}: \mathcal{G}_{\xi} \rightarrow \operatorname{Spec}(k(\xi))
$$

at points $\xi$ of a Noetherian Deligne-Mumford stack $S$ (cf. [28, §11], [38, App. B]). In particular, if $S$ is integral, there is a residual gerbe $\mathcal{G}$ at the generic point of $S$.

Proposition 2. Let $S$ be a regular integral Noetherian Deligne-Mumford stack with residual gerbe $\mathcal{G}$ at the generic point. Then the restriction map $\operatorname{Br}(S) \rightarrow \operatorname{Br}(\mathcal{G})$ is injective. Furthermore, if $\operatorname{dim} S=2$ then for any positive integer $n$, invertible in the local rings of an étale atlas of $S$, the residue maps fit into an exact sequence

$$
0 \rightarrow \operatorname{Br}(S)[n] \rightarrow \operatorname{Br}(\mathcal{G})[n] \rightarrow \bigoplus_{\xi \in S^{(1)}} H^{1}\left(\mathcal{G}_{\xi}, \mathbb{Z} / n \mathbb{Z}\right)
$$

where $S^{(1)}$ denotes the set of codimension 1 points of $S$.

Proof. For the inclusion $i: \mathcal{G} \rightarrow S$ the injectivity of $i^{*}: \operatorname{Br}(S) \rightarrow \operatorname{Br}(\mathcal{G})$ reduces by the Leray spectral sequence $H^{p}\left(S, R^{q} i_{*}\left(\mathbb{G}_{m, \mathcal{G}}\right)\right) \Rightarrow H^{p+q}\left(\mathcal{G}, \mathbb{G}_{m, \mathcal{G}}\right)$ and the identification of $\operatorname{coker}\left(\mathbb{G}_{m, S} \rightarrow i_{*} \mathbb{G}_{m, \mathcal{G}}\right)$ with the sheaf of divisors on $S$ [23, II.1 (2)] to the vanishing of $H^{1}\left(\mathcal{G}_{\xi}, \mathbb{Z}\right)$ for $\xi \in S^{(1)}$. The Leray spectral sequence for $(2.1)$ yields the exact sequence

$$
0 \rightarrow H^{1}(\operatorname{Spec}(k(\xi)), \mathbb{Z}) \rightarrow H^{1}\left(\mathcal{G}_{\xi}, \mathbb{Z}\right) \rightarrow H^{0}\left(\operatorname{Spec}(k(\xi)), R^{1} f_{\xi *} \mathbb{Z}\right)
$$

and we have $H^{1}(\operatorname{Spec}(k(\xi)), \mathbb{Z})=0$ (standard) and $R^{1} f_{\xi *} \mathbb{Z}=0$ by the identification with group cohomology, cf. [21, §4]. The rest of the argument is just as in the scheme case [7], [23].

2.2. Gerbes. Let $X$ be a Noetherian Deligne-Mumford stack, and let $n$ be a positive integer invertible in the local rings of an étale atlas of $X$. By a $\mathbb{G}_{m}$-gerbe, respectively $\mu_{n}$-gerbe on $X$ we mean a gerbe banded by the commutative algebraic group $\mathbb{G}_{m}$, respectively $\mu_{n}$. Recall (c.f. $[33, \S 4.2]$ ), this is an algebraic stack $G$ with a morphism to $X$, which étale locally admits sections, étale locally lets any pair of sections be identified, and which is equipped with compatible identifications of the automorphism groups of local sections with the invertible regular functions, respectively, $\mu_{n}$-valued functions. In the $\mu_{n}$ case, $G$ is also a Deligne-Mumford stack. 
A gerbe is classified uniquely up to isomorphism, compatible with the identification of automorphism groups of local objects, by a cohomology class in

$$
H^{2}\left(X, \mathbb{G}_{m}\right) \quad \text { or } \quad H^{2}\left(X, \mu_{n}\right)
$$

in the respective cases.

Proposition 3. Let $X$ be a Noetherian Deligne-Mumford stack and $n$ a positive integer invertible in the local rings of an étale atlas of $X$. Then the boundary map of nonabelian cohomology for the central exact sequence of groups

$$
1 \rightarrow \mu_{n} \rightarrow S L_{n} \rightarrow P G L_{n} \rightarrow 1
$$

associates to a sheaf of Azumaya algebras $\mathcal{A}$ on $X$ of index $n$ a $\mu_{n}$-gerbe

$$
G \rightarrow X
$$

and a locally free sheaf $E$ of rank $n$ on $G$ such that $E^{\vee} \otimes E$ is identified with the pullback of $\mathcal{A}$.

Proof. The class $\gamma \in H^{2}\left(X, \mu_{n}\right)$, the image of the class of $\mathcal{A}$ by the boundary map, is the class of the $\mu_{n}$-gerbe

$$
\left[P / S L_{n}\right],
$$

where $P \rightarrow X$ denote the $P G L_{n}$-torsor associated with $\mathcal{A}$ (cf. [23, §I.2]). The representation given by the inclusion $S L_{n} \rightarrow G L_{n}$ determines a vector bundle $E$ on $\left[P / S L_{n}\right]$ and an isomorphism of $E^{\vee} \otimes E$ with the pullback of $\mathcal{A}$.

Proposition 4. In the situation of Proposition 3, suppose that $\mathcal{A}$ is trivial in the Brauer group. Then there exists a line bundle $T$ on the $\mu_{n}$-gerbe $G$ such that the $\mu_{n}$-action on fibers is via the inclusion $\mu_{n} \rightarrow \mathbb{G}_{m}$.

Proof. By hypothesis, $\mathcal{A} \cong F^{\vee} \otimes F$ for some locally free sheaf $F$ of rank $n$ on $X$. The $P G L_{n}$-torsor $P \rightarrow X$ associated with $\mathcal{A}$ may then be described as the stack of projective trivializations $\mathbb{P}\left(\mathcal{O}_{X}^{n}\right) \cong \mathbb{P}(F)$, and the $\mu_{n}$-gerbe $G$ is $\left[P / S L_{n}\right]$. The stack of compatible linear maps $\mathcal{O}_{X}^{n} \rightarrow F$ descends (via standard descent along a torsor, cf. [44, Thm. 4.46]) to a line bundle on $G$ with the desired property.

2.3. Root stack. Let $S$ be a regular integral Noetherian Deligne-Mumford stack, $D \subset S$ an effective divisor, and $n$ a positive integer. The corresponding root stack will be denoted by $\sqrt[n]{(S, D)}([15, \S 2],[2$, App. B]). This is a Deligne-Mumford stack when $n$ is invertible in the local rings of an étale atlas of $S$. Above $D$ is the gerbe of the root stack [15, Def. 2.4.4], an effective divisor of $\sqrt[n]{(S, D)}$ which is a $\mu_{n}$-gerbe over $D$. The morphism $\sqrt[n]{(S, D)} \rightarrow S$ is flat, is ramified over $D$, and restricts to an isomorphism over $S \backslash D$.

Proposition 5. Let $S$ be a regular integral Noetherian Deligne-Mumford stack of dimension 2 and $D \subset S$ a regular closed substack of codimension 1. Then, for a positive integer $n$ invertible in the local rings of an étale atlas of $S$ and $n$-torsion Brauer class $\alpha \in \operatorname{Br}(S \backslash D)$ there exists a unique class

$$
\beta \in \operatorname{Br}(\sqrt{(S, D)})
$$

such that the restriction of $\beta$ to $S \backslash D$ is equal to $\alpha$.

Proof. The residue maps of $D$ and the gerbe of the root stack differ by a factor of $n$, so we have the result by the exact sequence of Proposition 2 applied to $\sqrt{(S, D)}$. 
2.4. Algebraic cycles. Most of the material here can be found in [8]. We recall that the triviality of the Brauer group of a proper smooth variety is connected with the triviality of the Chow group $\mathrm{CH}_{0}$ of 0 -cycles modulo rational equivalence upon extension of the base field.

Proposition 6. Let $k$ be an algebraically closed field, and $X$ a proper smooth algebraic variety over $k$. We let $\Delta \subset X \times X$ denote the diagonal and

$$
\mathrm{pr}_{1}, \mathrm{pr}_{2}: X \times X \rightarrow X
$$

the projections. For a field $L$ containing $k$ we let $X_{L}$ denote $X \times_{\operatorname{Spec}(k)} \operatorname{Spec}(L)$. The following conditions are equivalent:

(i) The degree map $\mathrm{CH}_{0}\left(X_{L}\right) \rightarrow \mathbb{Z}$ is an isomorphism for every field extension $L / k$.

(ii) The degree map $C H_{0}\left(X_{k(X)}\right) \rightarrow \mathbb{Z}$ is an isomorphism.

(iii) The class of $\Delta$ in $C H_{\operatorname{dim} X}(X \times X)$ is equal to a class of the form $\delta+\operatorname{pr}_{2}^{*} \omega$, where $\delta$ is a cycle supported on $D \times X$ for some closed $D \subset X$ of codimension 1 and $\omega$ is a 0 -cycle on $X$ of degree 1 .

Furthermore, the conditions imply the vanishing of the n-torsion in $\operatorname{Br}(X)$ for every integer $n$ invertible in $k$.

Proof. A proof can be found in [18, Prop. 1.4] but we sketch the argument for convenience. The implications (i) $\Rightarrow$ (ii) $\Rightarrow$ (iii) are clear, and (iii) $\Rightarrow$ (i) follows from $\left(\mathrm{pr}_{2}\right)_{*}\left(\operatorname{pr}_{1}^{*} \eta \cdot[\Delta]\right)=\eta$ for $\eta \in C H_{0}(X)$ and a similar identity after base change to $L$. Merkurjev has shown [32, Thm. 2.11] that conditions (i)-(iii) are equivalent to $k(X)$ having only trivial unramified elements for every cycle module $M$ in the sense of Rost [36]. Applying this to étale cohomology with values in $\mu_{n}$ (cf. [16]) yields $\operatorname{Br}(X)[n]=0$ for $n$ invertible in $k$.

For a proper algebraic variety $X$ over an algebraically closed field $k$, we say that there is a Chow decomposition of the diagonal for $X$ if condition (iii) holds for $X$. There is no loss of generality in taking $\omega$ in (iii) to be $\{x\}$ where $x$ is a $k$-point of $X$. (The argument of (iii) $\Rightarrow$ (i) is applicable and yields $\{x\}=\omega$ in $C H_{0}(X)$ when $x$ is a smooth point of $X$, not contained in $D$, using that $\{x\} \times X \rightarrow X \times X$ is a regular embedding; a singular point or point of $D$ may be expressed in $C H_{0}(X)$ as a linear combination of smooth points of $X \backslash D$.) The condition, to admit a Chow decomposition of the diagonal, also makes sense for Chow groups with coefficients in an arbitrary commutative ring.

Proposition 7. Let $k$ be an algebraically closed field, and $X$ a smooth projective algebraic variety over $k$. If $X$ is stably rational, then $X$ admits a Chow decomposition of the diagonal.

Proof. If $X$ is stably rational, then $X$ is retract rational [39]: there exist an integer $N$, nonempty open $U \subset X$ and $V \subset \mathbb{A}^{N}$, and morphisms $\varphi: U \rightarrow V$ and $\psi: V \rightarrow U$ such that $\psi \circ \varphi=\operatorname{id}_{U}$. By [18, Lem. 1.5], every smooth projective variety that is retract rational admits a Chow decomposition of the diagonal.

Proposition 8. Let $X$ be a proper three-dimensional algebraic variety over an algebraically closed field $k$ with only ordinary double point singularities and $\widetilde{X} \rightarrow X$ the standard resolution obtained by blowing up the singular points of $X$. Then there is a Chow decomposition of the diagonal for $\tilde{X}$ if and only if there is a Chow decomposition of the diagonal for $X$. 
Proof. Since pushforward of cycles respects rational equivalence, the forward implication is clear. The reverse implication follows from the fact that each exceptional divisor of the resolution is isomorphic to $\mathbb{P}^{1} \times \mathbb{P}^{1}$, and up to rational equivalence any 3-cycle in $\widetilde{X} \times \mathbb{P}^{1} \times \mathbb{P}^{1}$ may be written as the sum of a cycle supported over a divisor on $\widetilde{X}$ and the pullback of a 0 -cycle from $\mathbb{P}^{1} \times \mathbb{P}^{1}$.

\section{Chow DeComposition of the Diagonal in FAmilies}

The proof of the main theorem uses the following version of Voisin's result.

Theorem 9. Let $B$ be an algebraic variety over an uncountable algebraically closed field $k$ and $\pi: \mathcal{C} \rightarrow B$ a flat projective morphism with integral fibers. Let $R$ be the ring $\mathbb{Z}$ if $k$ has characteristic 0 , and $\mathbb{Z}[1 / p]$ if $k$ has characteristic $p>0$. If there exists a k-point $b_{0} \in B$ such that the fiber $\mathcal{C}_{b_{0}}$ does not admit a Chow decomposition of the diagonal with coefficients in $R$, then for very general $b \in B$ the fiber $\mathcal{C}_{b}$ does not admit a Chow decomposition of the diagonal with coefficients in $R$.

Proof. The set of $k$-points $b \in B$ such that the fiber $\mathcal{C}_{b}$ admits a Chow decomposition of the diagonal with coefficients in $R$ is a countable union of proper closed subvarieties of $B$. In characteristic 0 this is [18, Thm. 2.3, App. B]. An initial reduction step in loc. cit. lets us assume that $\pi$ admits a section $s: B \rightarrow \mathcal{C}$.

An argument valid in characteristic $p>0$ may be formulated using the formalism of Chow functors of [37]. We recall, rational equivalence of cycles may be formulated in terms of cycles on a product with $\mathbb{P}^{1}$; cf. $[22, \S 1.6]$. We fix a sufficiently ample invertible sheaf $\mathcal{O}(1)$ on $\mathcal{C}$, with which we measure degrees on the fibers of $\pi$; for degrees of cycles on the fibers of $\mathcal{C} \times{ }_{B} \mathcal{C} \rightarrow B$ and $\mathcal{C} \times{ }_{B} \mathcal{C} \times \mathbb{P}^{1} \rightarrow B$ we use $\mathcal{O}(1,1)$, respectively $\mathcal{O}(1,1,1)$. Let $r$ denote the relative dimension of $\mathcal{C}$ over $B$. For a positive integer $d$ we let $W_{d}$ be a scheme, quasi-projective over the projective bundle $\mathbb{P}\left(\pi_{*} \mathcal{O}(d)\right)$ of relative degree $d$ divisors $\mathcal{D} \subset \mathcal{C}$, equipped with effective relative cycles $\delta_{1}$ and $\delta_{2}$ on $\mathcal{D} \times{ }_{B} \mathcal{C}$ of (relative) dimension $r$ and degree at most $d p^{d}$ and $\varepsilon$ on $\mathcal{C} \times{ }_{B} \mathcal{C} \times \mathbb{P}^{1}$ of dimension $r+1$ and degree at most $d p^{d}$, such that:

(i) For every $w \in W_{d}$ and $z \in \mathbb{P}_{k(w)}^{1}$ the cycle $\left.\varepsilon\right|_{\{w\}}$ meets $\mathcal{C} \times{ }_{B} \mathcal{C} \times\{z\}$ properly.

(ii) Every $k$-point $b \in B$, divisor $D \in\left|\mathcal{O}_{\mathcal{C}_{b}}(d)\right|$, and triple of cycles satisfying (i) occur at some $k$-point of $W_{d}$.

(Using Hilbert-to-Chow morphisms, the normalization of a finite union of projective Hilbert schemes satisfies the conditions, ignoring the proper intersection condition, and by semicontinuity of fiber dimension the proper intersection condition determines an open subscheme.)

For effective relative cycles there are operations of addition and intersection with a relative Cartier divisor [37, (IV.4.15), (IV.15.2)]. The equality of cycles

$$
p^{d} \Delta+\delta_{1}+\left(\mathcal{C} \times{ }_{B} \mathcal{C} \times\{0\}\right) \cdot \varepsilon=p^{d}\left(\mathcal{C} \times{ }_{B} s(B)\right)+\delta_{2}+\left(\mathcal{C} \times{ }_{B} \mathcal{C} \times\{\infty\}\right) \cdot \varepsilon
$$

defines a Zariski closed subset

$$
V_{d} \subset W_{d}
$$

Indeed, given a scheme $T$ of finite type over $B$ and pair of effective relative cycles of $\mathcal{C} \times{ }_{B} T$, proper and equidimensional of dimension $r$ over $T$, the set of points of $T$ at which the cycles are equal is Zariski closed in $T$. This is because the support of a relative cycle over $T$ is universally open over $T$ [37, (IV.4.7)], thus the locus where the supports are equal is closed in $T$. Given two cycles over $T$ with the same support $Z$, in order to show that the locus of equality is closed it suffices by Noetherian induction 
to show: if every nonempty open $U \subset T$ has a point $u \in U$ at which the cycles are equal, then the cycles are equal at every point of $T$. Let us write $Z=Z_{1} \cup \cdots \cup Z_{\ell}$ (irreducible components). We may replace $T$ by its reduced subscheme, then we restrict our attention to open $U$ contained in the normal locus of $T$, such that for every $i$ both $Z_{i} \times_{T} U$ and $\left(Z_{i} \cap\left(Z_{1} \cup \cdots \cup \widehat{Z}_{i} \cup \cdots \cup Z_{\ell}\right)\right) \times_{T} U$ are flat over $U$. Then equality of cycles at a point $u \in U$ implies equality at the generic point of the component of $U$ containing $u$ (cf. [37, (IV.10.4)]). Now, as in [37, (IV.6.1)], equality of cycles at the generic point of every irreducible component of $T$ implies equality of cycles at all points of $T$.

We let $U_{d}$ denote the image of $V_{d}$ in $B$. We have $U_{d} \subset U_{d+1}$, and the $k$-points of the union

$$
\mathcal{U}:=\bigcup_{d=1}^{\infty} U_{d}
$$

are precisely the $k$-points $b \in B$ for which $\mathcal{C}_{b}$ admits a Chow decomposition of the diagonal with coefficients in $\mathbb{Z}[1 / p]$.

We are done if we can show that for any positive integer $d$, pointed smooth curve $(A, a)$, and morphism $A \rightarrow B$ such that the image of the generic point of $A$ lies in $U_{d}$, the image $b \in B$ of $a$ lies in $\mathcal{U}$. It suffices to do this under the further assumption that the restriction $A \backslash\{a\} \rightarrow B$ factors through $V_{d}$. Then there are corresponding effective cycles with $\mathbb{Z}[1 / p]$-coefficients, flat over $A \backslash\{a\}$, and by $[37$, (IV.10.5)] these specialize at $k$-points of $A \backslash\{a\}$ to the respective corresponding cycles $\delta_{1}, \delta_{2}, \varepsilon$. Equation (3.1) translates into an equality of cycles over $A \backslash\{a\}$. By applying the cycle-level specialization maps [22, Rmk. 2.3] and the commutativity of pairs of specialization maps up to rational equivalence [22, Thm. 2.4], we obtain $b \in \mathcal{U}$.

In combination with Proposition 8 we therefore have:

Corollary 10. Let $B$ be an algebraic variety over an uncountable algebraically closed field $k$ and $\mathcal{C} \rightarrow B$ a flat projective morphism of relative dimension 3 with integral fibers, generically smooth with $k$-point $b_{0} \in B$ such that the fiber $\mathcal{C}_{b_{0}}$ has only ordinary double point singularities. Let $\widetilde{\mathcal{C}}_{b_{0}} \rightarrow \mathcal{C}_{b_{0}}$ denote the standard resolution obtained by blowing up the singularities. If $\operatorname{Br}\left(\widetilde{\mathcal{C}}_{b_{0}}\right)[n] \neq 0$ for some integer $n$ invertible in $k$, then for very general $b \in B$ the fiber $\mathcal{C}_{b}$ is not stably rational.

In particular, assuming the characteristic of $k$ to be different from 2, we may apply Corollary 10 in case $\mathcal{C}_{b_{0}}$ is a conic bundle over a smooth projective rational surface such that

(i) The branch locus is a union $D_{1} \cup D_{2}$ where $D_{1}$ and $D_{2}$ are smooth curves meeting each other transversely.

(ii) The ramification data consists of degree 2 unramified covers of $D_{1}$ and $D_{2}$.

Indeed, a conic bundle model of $\mathcal{C}_{b_{0}}$ with smooth total space has branch locus the disjoint union of $D_{1}$ and $D_{2}$, and by [7, Prop. 3] (see also [17]) there is nontrivial 2-torsion in the unramified Brauer group of $\widetilde{\mathcal{C}}_{b_{0}}$.

\section{Construction}

4.1. Construction I: Brauer class on root stacks. We let $S$ be a smooth rational projective surface over an algebraically closed field $k$ of characteristic different from 2 . Let $D \subset S$ be a divisor of the form $D_{1} \cup D_{2}$ where $D_{1}$ and $D_{2}$ are smooth irreducible curves on $S$, each of positive genus. We assume that $D_{1}$ and $D_{2}$ meet transversely in 
some points $p_{1}, \ldots, p_{r}$. Following Artin and Mumford [7, Thm. 1], letting $U:=S \backslash D$, a 2-torsion element of the Brauer group

$$
\alpha \in \operatorname{Br}(U)
$$

gives rise to double covers of $D_{1}$ and $D_{2}$ étale away from the intersection points; these determine $\alpha$ uniquely. Moreover, étale double covers of $D_{1}$ and $D_{2}$ uniquely determine a 2-torsion element $\alpha \in \operatorname{Br}(U)$.

In the setting of $D=D_{1} \cup D_{2}$ on $S$ with given nontrivial étale double covers of $D_{1}$ and $D_{2}$, the root stack

$$
X:=\sqrt{(S, D)}
$$

has an ordinary double point singularity above each point $p_{i}$ and is otherwise smooth. Proposition 5 applied to $S \backslash\left\{p_{1}, \ldots, p_{r}\right\}$ shows that the class $\alpha$ is the restriction of a unique element

$$
\beta \in \operatorname{Br}\left(X^{\mathrm{sm}}\right) .
$$

We will show that $\beta$ extends to a class in $\operatorname{Br}(X)$. We start, following Lieblich [29], by producing an extension of $\beta$ to a smooth compactification of $X^{\mathrm{sm}}$. The smooth compactification that we use is the iterated root stack

$$
X^{\prime}:=\sqrt{\left(S,\left\{D_{1}, D_{2}\right\}\right)}
$$

introduced in [15, Def. 2.2.4], which differs from the root stack $X$ in that the stabilizer group above each point $p_{i}$ is $\mu_{2} \times \mu_{2}$ rather than $\mu_{2}$. The stack $X^{\prime}$ admits a morphism

$$
\tau: X^{\prime} \rightarrow X,
$$

restricting to an isomorphism over $X^{\mathrm{sm}}$. By Proposition 2, there is a unique class

$$
\beta^{\prime} \in \operatorname{Br}\left(X^{\prime}\right)
$$

restricting to $\beta \in \operatorname{Br}\left(X^{\mathrm{sm}}\right)$.

Given a $k$-point $p \in S$ we let $\mathcal{O}_{S, \bar{p}}$ denote the (strict) Henselization of the local ring of $S$ at $p$.

Lemma 11. For every $j$ the class $\alpha$ lies in the kernel of

$$
\operatorname{Br}(U) \rightarrow \operatorname{Br}\left(\operatorname{Spec}\left(\mathcal{O}_{S, \bar{p}_{j}}\right) \times_{S} U\right) .
$$

Proof. This is immediate from Proposition 2 and the fact that the degree 2 covers of $D_{1}$ and $D_{2}$ are étale.

Proposition 12. The element $\alpha$ is represented by a sheaf of Azumaya algebras $\mathcal{A}^{\prime}$ on $X^{\prime}$ of index 2.

Proof. It is a general fact for $C_{2}$-fields of characteristic different from 2, e.g., the function field $k(S)$, that a nontrivial 2-torsion Brauer group element is the class of a quaternion algebra; see [41, Thm. 5.7]. Since $X^{\prime}$ is Noetherian and regular of dimension 2, such a quaternion algebra is the restriction of a sheaf of Azumaya algebras.

Let $f: Z \rightarrow W$ be a finite-type morphism of separated Noetherian Deligne-Mumford stacks. Then there is a relative notion of coarse moduli space, introduced in [4], which determines a stack with representable morphism to $W$, through which $f$ factors. The relative moduli space is characterized by the standard sort of universal property, after base change to an étale atlas of $W$. In many cases $W$ already has this property; 
then we will say, $f$ is a relative moduli space. Root stacks are one important class of examples. The morphism $\tau$ above is also a relative moduli space.

Under a tameness hypothesis, satisfied by our assumption that the characteristic of $k$ is different from 2, a locally free coherent sheaf is obtained as pullback of a locally free coherent sheaf from a relative moduli space if and only if the relative stabilizer group actions on fibers at closed points are trivial [6, Thm. 10.3].

Proposition 13. The action of the diagonal $\mu_{2}$ in the stabilizer $\mu_{2} \times \mu_{2}$ of the closed point of $\operatorname{Spec}\left(\mathcal{O}_{S, \bar{p}_{j}}\right) \times_{S} X^{\prime}$ on the fiber of $\mathcal{A}^{\prime}$ is trivial.

Proof. By Lemma 11, for each $j$ the pullback of $\beta^{\prime}$ to $\operatorname{Spec}\left(\mathcal{O}_{S, \bar{p}_{j}}\right) \times_{S} X^{\prime}$ vanishes. So the $P G L_{2}$-representation coming from $\mathcal{A}^{\prime}$ of the stabilizer $\mu_{2} \times \mu_{2}$ at the closed point of $\operatorname{Spec}\left(\mathcal{O}_{S, \bar{p}_{j}}\right) \times_{S} X^{\prime}$ must lift to a $G L_{2}$-representation, which is well-defined up to twist by a character of $\mu_{2} \times \mu_{2}$. Thus we may take it to have the form of the direct sum of a trivial representation and a character. We claim that the character must be the composite

$$
\mu_{2} \times \mu_{2} \rightarrow \mu_{2} \rightarrow k^{\times}
$$

of the multiplication and the nontrivial character of $\mu_{2}$. Indeed, the criterion for a locally free coherent sheaf to be a pullback via

$$
X^{\prime} \rightarrow \sqrt{\left(S, D_{i}\right)}
$$

for $i \in\{1,2\}$, is nontrivial only on the gerbe of the root stack over $D_{3-i}$, and since $D_{3-i}$ is connected the criterion is satisfied everywhere if it is satisfied at one point. So the claim follows from the fact that $\alpha$ does not extend to the generic point of $D_{3-i}$.

Corollary 14. The sheaf of Azumaya algebras $\mathcal{A}^{\prime}$ is isomorphic to the pullback of a sheaf of Azumaya algebras $\mathcal{A}$ on $X$.

Proof. This follows by the criterion for pullback of a locally free coherent sheaf, applied to $\tau: X^{\prime} \rightarrow X$.

The class of $\mathcal{A}$ in $\operatorname{Br}(X)$ extends $\beta \in \operatorname{Br}\left(X^{\mathrm{sm}}\right)$ and will be denoted as well by $\beta$.

4.2. Construction II: Conic bundle. We have $G=\left[P / S L_{2}\right]$, the $\mu_{2}$-gerbe of Proposition 3 applied to the stack $X$ and the sheaf of Azumaya algebras $\mathcal{A}$, which, we may recall, is classified by the cohomology class

$$
\gamma \in H^{2}\left(X, \mu_{2}\right)
$$

coming from $\mathcal{A}$ via the boundary homomorphism of nonabelian cohomology.

Lemma 15. Suppose that $\widetilde{E}$ is a locally free sheaf of rank 2 on the $\mu_{2}$-gerbe $G$ with nontrivial generic stabilizer action. Then the sheaf of Azumaya algebras $\widetilde{\mathcal{A}}$ on $X$, defined uniquely up to isomorphism by the requirement that the pullback of $\widetilde{\mathcal{A}}$ to $G$ is isomorphic to $\widetilde{E}^{\vee} \otimes \widetilde{E}$, is Brauer equivalent to $\mathcal{A}$.

Proof. The representable morphism

$$
\left[P / S L_{2}\right] \rightarrow\left[P / G L_{2}\right]
$$

from the $\mu_{2}$-gerbe to the $\mathbb{G}_{m}$-gerbe identifies the categories of locally free coherent sheaves for which the generic stabilizer action is the scalar action by the inclusion $\mu_{2} \rightarrow \mathbb{G}_{m}$, respectively the identity morphism of $\mathbb{G}_{m}$; cf. [30, Lemma 3.5]. So there is a locally free sheaf $\widetilde{F}$ of rank 2 on $\left[P / G L_{2}\right]$ whose pullback to $G$ is isomorphic to 
$\widetilde{E}$. The associated principal $G L_{2}$-bundle of $\widetilde{F}$ may be identified with the principal $P G L_{2}$-bundle $\widetilde{P}$ associated with $\widetilde{\mathcal{A}}$, inducing

$$
\left[P / G L_{2}\right] \cong\left[\widetilde{P} / G L_{2}\right]
$$

and hence an equality of the corresponding Brauer classes.

Proposition 16. With $S, D, X=\sqrt{(S, D)}, \gamma \in H^{2}\left(X, \mu_{2}\right)$, and $G$ as above, let $\left(B, b_{0}\right)$ be a smooth pointed algebraic variety over $k$ and $\mathcal{D} \subset B \times S$ an irreducible smooth divisor such that $\mathcal{D} \cap\left(\left\{b_{0}\right\} \times S\right)=D$. We let $\mathcal{X}$ denote the root stack $\sqrt{(B \times S, \mathcal{D})}$. If

$$
\Gamma \in H^{2}\left(\mathcal{X}, \mu_{2}\right)
$$

is a class restricting to $\gamma$ under the inclusion of $X$ in $\mathcal{X}$, and we let

$$
\mathcal{G} \rightarrow \mathcal{X}
$$

denote a $\mu_{2}$-gerbe with class $\Gamma$, then for any locally free sheaf $\widetilde{\mathcal{E}}$ of rank 2 on $\mathcal{G}$ with nontrivial generic stabilizer action, the associated smooth conic fibration

$$
\widetilde{\mathcal{C}} \rightarrow \mathcal{X}
$$

characterized uniquely up to isomorphism by

$$
\mathcal{G} \times{ }_{\mathcal{X}} \widetilde{\mathcal{C}} \cong \mathbb{P}(\widetilde{\mathcal{E}}),
$$

has nontrivial stack structure at precisely two geometric points above every geometric point of $\mathcal{D}$. The blow-up

$$
B \ell_{\widetilde{\mathcal{C}}_{\text {stack }}} \widetilde{\mathcal{C}} \rightarrow \mathcal{X}
$$

of the locus with nontrivial stack structure is a flat family of genus 0 prestable curves whose geometric fibers are irreducible over points of $\mathcal{X} \backslash \mathcal{D}$ and chains of three irreducible components over points of $\mathcal{D}$. Collapsing the "middle component" of the three-component chains yields a flat family of genus 0 prestable curves which is isomorphic to the base-change by $\mathcal{X} \rightarrow B \times S$ of a standard conic bundle

$$
\mathcal{C} \rightarrow B \times S \text {. }
$$

We recall, a prestable curve is a connected proper nodal curve; cf. [13, Defn. 2.1].

Proof. Since $\mathcal{D}$ is connected, it suffices to check the structure of the locus with stack structure above a single point. By hypothesis, $\mathcal{G} \times \mathcal{X} X$ may be identified with $G$. By combining Lemma 15 applied to $\left.\widetilde{\mathcal{E}}\right|_{G}$ with the analysis of Proposition 13 applied to the associated sheaf of Azumaya algebras, pulled back to $X^{\prime}$, we see that $\widetilde{\mathcal{C}} \rightarrow \mathcal{X}$ has fibers isomorphic to $\left[\mathbb{P}^{1} / \mu_{2}\right]$ for nontrivial $\mu_{2}$-action on $\mathbb{P}^{1}$ over the gerbe of the root stack $\mathcal{X}$. The blow-up is smooth, so flatness follows from the fibers being of constant dimension, and the geometric fibers are as claimed. The collapsing of the middle components is a standard construction, achievable for instance by formation of Proj of the sum of the direct images of powers of the relative anticanonical sheaf; cf. [27, §5.2]. Since the normal bundle to $\widetilde{\mathcal{C}}^{\text {stack }}$ in $\widetilde{\mathcal{C}}$ has scalar stabilizer action, we may observe that the stabilizer action on singular fibers becomes trivial after collapsing the middle components, and hence $\mathcal{C}$ may be obtained by flat descent along $\mathcal{X} \rightarrow B \times S$. 
4.3. Construction III: Elementary transform. We will obtain a locally free sheaf $\widetilde{\mathcal{E}}$ as in Proposition 16 using deformation theory. The strategy is to start with the locally free sheaf $E$ on $G$ of Proposition 3 and apply a standard modification, called elementary transform, to produce a locally free sheaf $\widetilde{E}$ of rank 2 on $G$, which satisfies a deformation-theoretic condition to extend to a locally free sheaf $\widetilde{\mathcal{E}}$.

The locally free sheaf $E$ of Proposition 3 has the property that $E^{\vee} \otimes E$ descends to the sheaf of Azumaya algebras $\mathcal{A}$ on $X$. In this and similar situations we will also use the notation $E^{\vee} \otimes E$ for the corresponding locally free sheaf on $X$.

The deformation theory of locally free coherent sheaves with given determinant is recalled, e.g., in [35]. The space of obstructions for $E$ is the group

$$
\operatorname{ker}\left(H^{2}\left(X, E^{\vee} \otimes E\right) \rightarrow H^{2}\left(X, \mathcal{O}_{X}\right)\right),
$$

kernel of the trace homomorphism. We may work on $X^{\prime}$, since $X$ is a relative coarse moduli space of $X^{\prime}$, hence $R^{i} \tau_{*} \mathcal{F}^{\prime}=0$ for every quasicoherent sheaf $\mathcal{F}^{\prime}$ on $X^{\prime}$ and $i>0$ (cf. $[3, \S 3]$ ). There is a gerbe $G^{\prime}$, fitting into a fiber diagram:

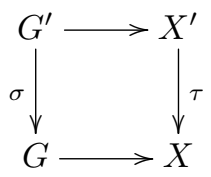

Letting

$$
E^{\prime}:=\sigma^{*} E,
$$

we have

$$
H^{i}\left(X, E^{\vee} \otimes E\right) \cong H^{i}\left(X^{\prime}, E^{\prime \vee} \otimes E^{\prime}\right)
$$

Since $X^{\prime}$ is smooth, tame, with projective coarse moduli space, there is Serre duality with the standard dualizing sheaf $K=K_{X^{\prime}}([34, \S 2]$, [14, App. B]). Furthermore, since the rank 2 of $E$ is invertible in $k$, the trace homomorphism admits a canonical splitting. Combining these facts, we identify the space of obstructions with the dual of

$$
H^{0}\left(X^{\prime},\left(E^{\prime \vee} \otimes E^{\prime}\right)_{0} \otimes K\right):=H^{0}\left(X^{\prime}, \operatorname{ker}\left(E^{\prime \vee} \otimes E^{\prime} \otimes K \rightarrow K\right)\right) .
$$

The kernel, here and below, is denoted with subscript 0 .

Let $C$ be a smooth irreducible orbifold curve on $X$, contained entirely in $X^{\mathrm{sm}}$, let $\left.E\right|_{C}$ denote by abuse of notation the restriction of $E$ to $G \times_{X} C$, and let

$$
\left.0 \rightarrow R \rightarrow E\right|_{C} \rightarrow Q \rightarrow 0
$$

be an exact sequence of coherent sheaves on $G \times_{X} C$, where $Q$ and $R$ are locally free of rank 1 . Then we define $\widetilde{E}$ to be the kernel of the composite of restriction to $G \times{ }_{X} C$ and the map to $Q$ from (4.1), so that we have the exact sequence

$$
0 \rightarrow \widetilde{E} \rightarrow E \rightarrow Q \rightarrow 0 .
$$

Proposition 17. There exist such an orbifold curve $C$ and such an exact sequence (4.1) of locally free coherent sheaves on $G \times_{X} C$, that the space of obstructions for the elementary transform $\widetilde{E}$ vanishes.

Proof. We may work on $X^{\prime}$ with

$$
\widetilde{E}^{\prime}:=\sigma^{*} \widetilde{E}
$$

and show that

$$
H^{0}\left(X^{\prime},\left(\widetilde{E}^{\prime \vee} \otimes \widetilde{E}^{\prime}\right)_{0} \otimes K\right)=0
$$


The argument follows closely that of [20] (written in the language of sheaves of Azumaya algebras rather than vector bundles on gerbes).

There is the exact sequence

$$
0 \rightarrow E^{\prime \vee} \rightarrow \widetilde{E}^{\prime \vee} \rightarrow Q^{\vee}(C) \rightarrow 0
$$

obtained by identifying $\operatorname{Ext}^{1}\left(Q, \mathcal{O}_{G^{\prime}}\right)$ with $Q^{\vee}(C)$. There are standard exact sequences involving the intersection $F^{\prime}$ of

in $\widetilde{E}^{\prime \vee} \otimes E^{\prime}$ :

$$
E^{\prime \vee} \otimes E^{\prime} \quad \text { and } \quad \widetilde{E}^{\prime \vee} \otimes \widetilde{E}^{\prime}
$$

$$
\begin{gathered}
0 \rightarrow F^{\prime} \rightarrow E^{\prime \vee} \otimes E^{\prime} \rightarrow R^{\vee} \otimes Q \rightarrow 0, \\
0 \rightarrow F^{\prime} \rightarrow \widetilde{E}^{\prime \vee} \otimes \widetilde{E}^{\prime} \rightarrow Q^{\vee}(C) \otimes R \rightarrow 0,
\end{gathered}
$$

giving rise to similar exact sequences involving the kernels of the trace map

$$
F_{0}^{\prime}, \quad\left(E^{\prime \vee} \otimes E^{\prime}\right)_{0}, \quad\left(\widetilde{E}^{\prime \vee} \otimes \widetilde{E}^{\prime}\right)_{0} .
$$

Now it suffices to show:

(i) There exist a finite set of points $\left\{x_{1}, \ldots, x_{n}\right\}$ of $U$ and points

$$
r_{1} \in \mathbb{P}\left(E_{x_{1}}\right), \quad \ldots, \quad r_{n} \in \mathbb{P}\left(E_{x_{n}}\right)
$$

such that for $C$ passing through $x_{1}, \ldots, x_{n}$ and exact sequence (4.1) such that $\left[R_{x_{i}}\right]=r_{i}$ for all $i$, the map on global sections of

$$
\left(E^{\prime \vee} \otimes E^{\prime}\right)_{0} \otimes K \rightarrow R^{\vee} \otimes Q \otimes K
$$

is injective.

(ii) With points $x_{1}, \ldots, x_{n}$ and $r_{1}, \ldots, r_{n}$ as in (i) and any choice of $C$ passing through $x_{1}, \ldots, x_{n}$, there exists an exact sequence (4.1) with $\left[R_{x_{i}}\right]=r_{i}$ for all $i$ and $R$ of arbitrarily negative degree.

Notice, then, $H^{0}\left(X^{\prime}, F_{0}^{\prime} \otimes K\right)=0$ and $H^{0}\left(C, Q^{\vee}(C) \otimes R \otimes K\right)=0$, and we conclude that $H^{0}\left(X^{\prime},\left(\widetilde{E}^{\prime \vee} \otimes \widetilde{E}^{\prime}\right)_{0} \otimes K\right)$ vanishes, as desired.

For (i), we may fix a trivialization of $K$ on some nonempty open $U_{1} \subset U$. For nonzero $s \in H^{0}\left(X^{\prime},\left(E^{\prime \vee} \otimes E^{\prime}\right)_{0} \otimes K\right)$ and $x \in U_{1}$ such that $s$ is nonzero in the fiber over $x$, we identify $s$ (using the trivialization of $K$ ) with a trace zero endomorphism of $E_{x}^{\prime}$. For any one-dimensional subspace $R_{x} \subset E_{x}^{\prime}$ which is not an eigenspace, $s$ maps to a nonzero element of $\operatorname{Hom}\left(R_{x}, E_{x}^{\prime} / R_{x}\right)$. Since $H^{0}\left(X^{\prime},\left(E^{\prime \vee} \otimes E^{\prime}\right)_{0} \otimes K\right)$ is finite dimensional, some finite collection of points and subspaces will satisfy (i).

For (ii) we apply Tsen's theorem, which gives the vanishing of the restriction of $\beta$ to $C$. So by Proposition 4 there is a line bundle $T$ on $G \times{ }_{X} C$ such that the generic stabilizer action is nontrivial. Then $E \otimes T^{\vee}$ descends to a vector bundle

$$
N \rightarrow C \text {, }
$$

and we obtain a sequence (4.1) from a similar sequence involving the vector bundle $N$ by tensoring with $T$. Notice, to give such a sequence is the same as to give a section of

$$
\mathbb{P}(N) \rightarrow C,
$$

which, being a representable projective morphism, is the same as to give a section of the restriction of (4.2) to a nonempty open substack of $C$. We choose $V \subset C$ that contains all the points $x_{1}, \ldots, x_{n}$, avoids all the orbifold points, and has the property that $\left.N\right|_{V}$ is trivial. A choice of trivialization identifies sections of (4.2) with maps 
$C \rightarrow \mathbb{P}^{1}$, which may be chosen to map $x_{i}$ to the point corresponding to $r_{i}$ for every $i$ and to have arbitrarily high degree.

\section{Proof of the main theorem}

Let the notation be as in Theorem 1 . By hypothesis, $\mathcal{M}$ contains a point corresponding to an étale degree 2 cover, nontrivial over every irreducible component of a reducible nodal curve with smooth irreducible compoments. We take, initially, this as the pointed variety $\left(B, b_{0}\right)$. For simplicity of notation we write $D=D_{1} \cup D_{2}$; the case of more than 2 irreducible components creates no additional difficulty since the criterion applied in the proof of Corollary 14 is local in nature, hence for $D=D_{1} \cup \cdots \cup D_{\ell}$ $(\ell>2)$ may be checked over $p \in D_{i} \cap D_{i^{\prime}}\left(i \neq i^{\prime}\right)$ by applying the reasoning of $\S 4.1$ to $D_{i}$ and $D_{i^{\prime}}$ after discarding $\bigcup_{j \notin\left\{i, i^{\prime}\right\}} D_{j}$.

The covering of $D$ determines a nontrivial 2-torsion Brauer group element $\alpha \in$ $\operatorname{Br}(S \backslash D)$. By Corollary 14, the class $\alpha$ pulls back and extends to the class $\beta \in \operatorname{Br}(X)$ of a sheaf of Azumaya algebras $\mathcal{A}$ of index 2 on the root stack $X=\sqrt{(S, D)}$. Now we fix a $\mu_{2}$-gerbe $G \rightarrow X$ and locally free coherent sheaf $E$ on $G$ as in Proposition 3 and let $\gamma \in H^{2}\left(X, \mu_{2}\right)$ be the class of $G$.

By Proposition 17, there is an elementary transform $\widetilde{E}$ of $E$ with vanishing obstruction for the deformation theory of locally free coherent sheaf with given determinant.

We let $\mathcal{D} \subset B \times S$ be the divisor corresponding to the linear system underlying $B$, which is smooth over a neighborhood of $b_{0}$ in $B$ by the hypothesis on base locus, and let $\mathcal{X}$ denote the root stack $\sqrt{(B \times S, \mathcal{D})}$. By the proper base change theorem for tame Deligne-Mumford stacks [1, App. A] applied to $\mathcal{X} \rightarrow B$, we may replace $B$ by an étale neighborhood of $b_{0}$ in such a way that the class $\gamma$ is the restriction of a class

$$
\Gamma \in H^{2}\left(\mathcal{X}, \mu_{2}\right) .
$$

We denote an associated $\mu_{2}$-gerbe by

$$
\mathcal{G} \rightarrow \mathcal{X}
$$

Lemma 18. The restriction map $\operatorname{Pic}(\mathcal{X}) \rightarrow \operatorname{Pic}(X)$ is surjective.

Proof. By the description of the Picard group of a root stack [15, §3.1], for a given line bundle $L$ on $X$ either $L$ or its twist by the divisor of the gerbe of root stack is pulled back from $X$. Since every line bundle on $X=\left\{b_{0}\right\} \times X$ is the restriction of a line bundle on $B \times X$, we have the result.

We apply Lemma 18 to the line bundle on $X$ whose pullback to $G$ is equal to $\operatorname{det}(\widetilde{E})$ to obtain a line bundle on $\mathcal{X}$ whose pullback to $\mathcal{G}$ restricts to $\operatorname{det}(\widetilde{E})$ on $G$. Now the deformation theory of locally free coherent sheaves with given determinant (which in our setting makes use of the Grothendieck existence theorem for tame DeligneMumford stacks of [5, App. A]), gives, after replacing $B$ by an étale neighborhood of $b_{0}$, the existence of a locally free coherent sheaf $\widetilde{\mathcal{E}}$ on $\mathcal{G}$ extending $\widetilde{E}$.

Proposition 16, applied to $\widetilde{\mathcal{E}}$, yields a standard conic bundle $\mathcal{C} \rightarrow B \times S$ whose restriction over $\left\{b_{0}\right\} \times S$ has an ordinary double point singularity over every point of $D_{1} \cap D_{2}$. In case the corresponding cover of $D$ differs from $\mathcal{M}$, elementary transform of $E$ along a smooth curve through some of the nodes of $D$ modifies the cover over those nodes. (The preimage of this curve in $X^{\prime}$ is nodal; the requisite quotient of the restriction of $E$ arises as the push-forward of an invertible sheaf from its normalization.) We conclude by applying Corollary 10 to the family $\mathcal{C} \rightarrow B$. 


\section{REFERENCES}

[1] D. Abramovich, A. Corti, and A. Vistoli, Twisted bundles and admissible covers, Comm. Algebra 31 (2003), 3547-3618.

[2] D. Abramovich, T. Graber, and A. Vistoli, Gromov-Witten theory of Deligne-Mumford stacks, Amer. J. Math. 130 (2008), 1337-1398.

[3] D. Abramovich, M. Olsson, and A. Vistoli, Tame stacks in positive characteristic, Ann. Inst. Fourier (Grenoble) 58 (2008), 1057-1091.

[4] D. Abramovich, M. Olsson, and A. Vistoli, Twisted stable maps to tame Artin stacks, J. Algebraic Geom. 20 (2011), 399-477.

[5] D. Abramovich and A. Vistoli, Compactifying the space of stable maps, J. Amer. Math. Soc. 15 (2002), 27-75.

[6] J. Alper, Good moduli spaces for Artin stacks, Ann. Inst. Fourier (Grenoble) 63 (2013), 23492402.

[7] M. Artin and D. Mumford, Some elementary examples of unirational varieties which are not rational, Proc. London Math. Soc. (3) 25 (1972), 75-95.

[8] A. Auel, J.-L. Colliot-Thélène, and R. Parimala, Universal unramified cohomology of cubic fourfolds containing a plane, to appear in Brauer groups and obstruction problems: moduli spaces and arithmetic (Palo Alto, CA, 2013).

[9] A. Beauville, Variétés de Prym et jacobiennes intermédiaires, Ann. Sci. École Norm. Sup. (4) 10 (1977), 309-391.

[10] A. Beauville, Le groupe de monodromie des familles universelles d'hypersurfaces et d'intersections complètes, Complex analysis and algebraic geometry (Göttingen, 1985), Lecture Notes in Math. 1194, Springer, Berlin, 1986, pp. 8-18.

[11] A. Beauville, A very general quartic double fourfold or fivefold is not stably rational, Algebraic Geometry, to appear.

[12] A. Beauville, A very general sextic double solid is not stably rational, arXiv:1411.7484.

[13] K. Behrend and Yu. Manin, Stacks of stable maps and Gromov-Witten invariants, Duke Math. J. 85 (1996), 1-60.

[14] U. Bruzzo and F. Sala, Framed sheaves on projective stacks, Adv. Math. 272 (2015), 20-95.

[15] C. Cadman, Using stacks to impose tangency conditions on curves, Amer. J. Math. 129 (2007), 405-427.

[16] J.-L. Colliot-Thélène, Birational invariants, purity and the Gersten conjecture, in K-theory and algebraic geometry: connections with quadratic forms and division algebras (Santa Barbara, CA, 1992), Proc. Sympos. Pure Math., 58, Part 1, Amer. Math. Soc., Providence, RI, 1995, pp. 1-64.

[17] J.-L. Colliot-Thélène and M. Ojanguren, Variétés unirationnelles non rationnelles: au-delà de l'exemple d'Artin et Mumford, Invent. Math. 97 (1989), no. 1, 141-158.

[18] J.-L. Colliot-Thélène and A. Pirutka, Hypersurfaces quartiques de dimension 3: non rationalité stable, Ann. Sci. École Norm. Sup. (4), to appear.

[19] J.-L. Colliot-Thélène and A. Pirutka, Revêtements cycliques qui ne sont pas stablement rationnels, arxiv:1506.00420.

[20] A. J. de Jong, The period-index problem for the Brauer group of an algebraic surface, Duke Math. J. 123 (2004), 71-94.

[21] P. Deligne and D. Mumford, The irreducibility of the space of curves of given genus, Inst. Hautes Études Sci. Publ. Math. 36 (1969), 75-109.

[22] W. Fulton, Intersection Theory, 2nd ed., Springer, Berlin, 1998.

[23] A. Grothendieck, Le groupe de Brauer, I-III, in Dix exposés sur la cohomologie des schémas, Advanced Studies in Pure Math. 3, North-Holland, Amsterdam, 1968, pp. 46-188.

[24] B. Hassett, A. Kresch, and Y. Tschinkel, On the moduli of degree 4 Del Pezzo surfaces, to appear in Development of moduli theory (Kyoto, 2013).

[25] V. A. Iskovskikh, On the rationality problem for conic bundles, Duke Math. J. 54 (1987), 271294.

[26] J. Kollár, Nonrational hypersurfaces, J. Amer. Math. Soc. 8 (1995), 241-249.

[27] A. Kresch, Flattening stratification and the stack of partial stabilizations of prestable curves, Bull. London Math. Soc. 45 (2013), 93-102.

[28] G. Laumon and L. Moret-Bailly, Champs Algébriques, Springer, Berlin, 2000.

[29] M. Lieblich, Period and index in the Brauer group of an arithmetic surface, J. Reine Angew. Math. 659 (2011), 1-41. 
[30] M. Lieblich, On the ubiquity of twisted sheaves, in F. Bogomolov, B. Hassett, Y. Tschinkel (eds.), Birational geometry, rational curves, and arithmetic, Springer, New York, 2013, pp. 205-227.

[31] M. Mella, On the unirationality of 3-fold conic bundles, arXiv:1403.7055.

[32] A. Merkurjev, Unramified elements in cycle modules, J. London Math. Soc. (2) 78 (2008), 51-64.

[33] J. S. Milne, Étale Cohomology, Princeton Univ. Press, Princeton, NJ, 1980.

[34] F. Nironi, Grothendieck duality for Deligne-Mumford stacks, arXiv:0811.1955.

[35] K. O'Grady, Moduli of vector bundles on surfaces, in Algebraic Geometry (Santa Cruz, 1995), Proc. Symposia Pure Math. 62, Part 1, Amer. Math. Soc., Providence, RI, 1997, pp. 101-126.

[36] M. Rost, Chow groups with coefficients, Doc. Math. 1 (1996), 319-393.

[37] D. Rydh, Families of cycles and the Chow scheme, Ph. D. thesis, KTH, Stockholm, 2008.

[38] D. Rydh, Étale dévissage, descent and pushouts of stacks, J. Algebra 331 (2011), 194-223.

[39] D. J. Saltman, Retract rational fields and cyclic Galois extensions, Israel Math. J. 47 (1984), $165-215$.

[40] V. G. Sarkisov, Birational automorphisms of conic bundles, Izv. Akad. Nauk SSSR Ser. Mat. 44 (1980), 918-945.

[41] V. G. Sarkisov, On conic bundle structures, Izv. Akad. Nauk SSSR Ser. Mat. 46 (1982), 371-408.

[42] V. V. Shokurov, Prym varieties: theory and applications, Izv. Akad. Nauk SSSR Ser. Mat. 47 (1983), 785-855.

[43] B. Totaro, Hypersurfaces that are not stably rational, arXiv:1502.04040.

[44] A. Vistoli, Grothendieck topologies, fibered categories and descent theory, in Fundamental algebraic geometry, Math. Surveys Monogr. 123, Amer. Math. Soc., Providence, RI, 2005, pp. $1-104$.

[45] C. Voisin, Unirational threefolds with no universal codimension 2 cycle, Invent. Math., to appear.

Department of Mathematics, MS 136, Rice University, Houston, TX 77005, USA

Current address: Department of Mathematics, Brown University, Box 1917, 151 Thayer Street,

Providence, RI 02912, USA

E-mail address: bhassett@math.brown.edu

Institut für Mathematik, Universität Zürich, Winterthurerstrasse 190, CH-8057 Zürich, SWITZERLAND

E-mail address: andrew.kresch@math.uzh.ch

Courant Institute, 251 Mercer Street, New York, NY 10012, USA and Simons Foundation, 160 Fifth Avenue, New York, NY 10010, USA

E-mail address: tschinkel@cims.nyu.edu 